\title{
An Underwater Acoustic Target Recognition Method Based on Spectrograms with Different Resolutions
}

\author{
Xinwei Luo* ${ }^{*}$, Minghong Zhang, Ting Liu, Ming Huang and Xiaogang Xu \\ Key Laboratory of Underwater Acoustic Signal Processing of Ministry of Education, Southeast University, \\ Nanjing 210096, China; 220200790@seu.edu.cn (M.Z.); 220200789@seu.edu.cn (T.L.); \\ 220200907@seu.edu.cn (M.H.); 220201070@seu.edu.cn (X.X.) \\ * Correspondence: luoxinwei@seu.edu.cn
}

check for updates

Citation: Luo, X.; Zhang, M.; Liu, T.; Huang, M.; Xu, X. An Underwater Acoustic Target Recognition Method Based on Spectrograms with Different Resolutions. J. Mar. Sci. Eng. 2021, 9, 1246. https://doi.org/10.3390/ jmse 9111246

Academic Editor: Dracos Vassalos

Received: 7 October 2021

Accepted: 9 November 2021

Published: 10 November 2021

Publisher's Note: MDPI stays neutral with regard to jurisdictional claims in published maps and institutional affiliations.

Copyright: (c) 2021 by the authors. Licensee MDPI, Basel, Switzerland. This article is an open access article distributed under the terms and conditions of the Creative Commons Attribution (CC BY) license (https:// creativecommons.org/licenses/by/ $4.0 /)$.

\begin{abstract}
This paper focuses on the automatic target recognition (ATR) method based on shipradiated noise and proposes an underwater acoustic target recognition (UATR) method based on ResNet. In the proposed method, a multi-window spectral analysis (MWSA) method is used to solve the difficulty that the traditional time-frequency (T-F) analysis method has in extracting multiple signal characteristics simultaneously. MWSA generates spectrograms with different T-F resolutions through multiple window processing to provide input for the classifier. Because of the insufficient number of ship-radiated noise samples, a conditional deep convolutional generative adversarial network (cDCGAN) model was designed for high-quality data augmentation. Experimental results on real ship-radiated noise show that the proposed UATR method has good classification performance.
\end{abstract}

Keywords: underwater acoustic target recognition; ship-radiated noise; time-frequency analysis; generative adversarial network; ResNet

\section{Introduction}

Underwater acoustic target recognition (UATR) is a kind of information processing technology that recognizes categories of targets through ship-radiated noise and the underwater acoustic echo received by SONAR. UATR has been widely used in human ocean activities such as fishing, marine search and rescue, seabed exploration, resource exploitation, and so on. It also provides an important decision-making basis for maritime military activities [1-3]. In the research related to UATR, the feature extraction method, the management of the sample set, and the design of the classifier are the critical research topics.

Ship-radiated noise has the property of short-time stability, so power spectrum analysis is an effective feature extraction method for ship-radiated noise. Power spectrum analysis converts signal energy from a complex distribution in the time domain to a relatively simple distribution in the frequency domain. The power spectrum is a stable expression of the ship-radiated noise signal, which can be used as a good classification feature. In Refs. [4,5], the power spectrum is used as the input of the classifier to achieve a good classification of ship targets. Auditory-based models are also used for feature extraction in underwater acoustic signals. Mel-frequency cepstrum coefficient (MFCC) is a spectrum feature designed based on human auditory characteristics that has been widely used in feature extraction from audio data, but has also been used in feature extraction from ship-radiated noise signals. Lanyue Zhang et al. [6] used MFCC, first-order differential MFCC, and second-order differential MFCC to design the feature extraction method for ship-radiated noise. Ref. [7] used MFCCs to extract the features of underwater acoustic signals as the input of the classifier. The auditory model is designed to simulate the receiving characteristics of the human ear, and it has a good effect on speech signal processing. However, the auditory model is not good at distinguishing high-frequency signals, which reduces the ability to extract high-frequency features from ship-radiated noise signals. To obtain the time-varying characteristics of the signal, time-frequency analysis is also 
a common feature extraction method for ship-radiated noise. The spectrogram of shipradiated noise signals can be obtained by short-time Fourier transform (STFT), which is also called low-frequency analysis and recording (LOFAR). Ref. [8] designed a deep learning recognition method based on time-domain data and the LOFAR spectrum to classify civil ships, large ships, and ferries. Wavelet analysis is also often used to extract features of underwater acoustic signals to obtain energy distributions with different time-frequency resolutions in the same spectrogram. Ref. [9] used wavelet analysis to extract features from underwater acoustic signals. However, the above feature extraction method obtains the target features through the model with a set of parameters, which lacks adaptability to different types of features.

The classifier classifies the samples based on the extracted signal features. Traditional classifier models include linear discriminant analysis (LDA), Gaussian mixture models (GMM) [10], support vector machines (SVM) [11], etc. However, in the classification of ship-radiated noise signals, the traditional classifier has many limitations. In the ocean, the complex environmental noise, the low SNR of underwater acoustic signals, and the interference of other ship-radiated noise make it difficult for the traditional classifier to obtain a good recognition effect. Compared with traditional methods, the introduction of artificial neural networks (ANN) significantly enhances the ability of the classifier. MLP [12], BP [13], CNN [14], and other networks are used to construct underwater acoustic signal classifiers. With the increase of network layers, the classification ability of deep neural networks becomes stronger and stronger. Deep neural networks (DNN) have been widely used in underwater acoustic signal classification. Ref. [4] extracts underwater acoustic signal features based on an RBM self-encoder and uses a BP classifier to obtain better recognition results than traditional recognition methods. Ref. [15] compares the performance of various classification networks in audio signals. AlexNet [16] has a clear structure and few training parameters, but the relatively simple network structure makes it unable to reach a high level of accuracy. The VGG network [17] has good classification results, but the deeper network structure makes the training long. Ref. [18] designed a network structure based on DenseNet, which has good classification results for underwater acoustic signals with different signal-to-noise ratios. However, DenseNet training requires a lot of memory space and has high requirements for the training environment. Ref. [19] proposed a ResNet model. Its residual module can effectively solve the problems of gradient explosion and gradient disappearance in DNNs, and has high training efficiency. It can also effectively shorten the training time under the condition of high accuracy.

DNNs generally need a large amount of data to train network parameters. Through training with a large number of samples, the characteristics of different categories can be fully extracted and the problem of overfitting can be effectively reduced. However, in practice, it is often impossible to obtain a large number of different categories of shipradiated noise signals, and some data augmentation methods are usually needed to expand the sample set. Data augmentation is mainly used to expand the training data set, diversify the data set as much as possible, and make the training model have strong generalization ability. Traditional data augmentation methods are mainly applied to the expansion of image sample sets. Data sets are expanded through rotation, scaling, shearing, and other operations of images, so as to train networks with good robustness [20]. However, the position of pixels in the spectrogram has the meaning of time and frequency, so rotation, cutting, and other operations cannot effectively generate new samples. With the development of machine learning, generative adversarial networks (GAN) [21] have received more and more attention and have been studied and applied in data augmentation. Some improved GANs have also been proposed. Mirza et al. proposed conditional GANs (CGAN) [22], adding tag data to both the generator and discriminator, and controlling input tags to control the category of generated samples. Alec Radford et al. proposed the deep convolutional GAN(DCGAN) model [23], which brings the structure of the convolutional neural network into GAN and makes many improvements to the original GAN, achieving a good generation effect. 
This paper proposes a UATR method based on ResNet, which has the following characteristics. (1) In UATR, a multi-window spectral analysis method is proposed, which can simultaneously extract spectrograms of different resolutions as classification samples. (2) Based on the advantages of CGANs and DCGANs, a conditional deep convolutional GAN (cDCGAN) model is proposed, which has achieved good results in data augmentation. Experimental results based on the ShipsEar database [24] show that the classification accuracy of the proposed method reaches $96.32 \%$. Accordingly, the classification accuracy of the GMM based classifier proposed in Ref. [24] is 75.4\%. The classification accuracy of the method based on the restricted Boltzmann machine (RBM) proposed in Ref. [4] is $93.17 \%$. The method proposed in Ref. [5] is based on a DNN classifier and uses the combined features of the power spectrum and the DEMON spectrum, achieving a classification accuracy of $92.6 \%$. The classifier proposed in Ref. [25] is constructed based on ResNet- 18 . The classifier adopts three features of Log Mel (LM), MFCC, the composition of chroma, contrast, Tonnetz, and zero-cross ratio (CCTZ); its classification accuracy reaches $94.3 \%$. The proposed method achieves the best performance in classification accuracy of the above UATRs.

In Section 2, the structure and implementation of the UATR are proposed and the feature extraction, data augmentation, and classification modules in the UATR are designed and discussed. In Section 3, the ShipsEar database is used to build a sample set, and the performance of the proposed UATR method is tested through experiments. Section 4 summarizes the article.

\section{The Framework and Implementation of UATR}

UATR usually consists of a feature extraction module and classifier module. Conventional classification features of ship-radiated noise include the power spectrum, MFCCs, GFCCs, and the LOFAR spectrum. The LOFAR graph is a common feature in engineering, since it has good time-frequency analysis ability and can be calculated quickly based on STFT. Since the STFT method needs to set parameters to obtain a different resolution, how to set parameters to adapt to the extraction of different signal components has always been a problem. In this paper, a multi-window spectral analysis method is designed. MWSA performs multiple STFT processing on a piece of data to generate multiple LOFAR images of the same size with different resolutions, which improves the ability to extract features from the original signal.

Classifiers based on DNNs are now widely used in underwater acoustic target classification and recognition. The training of DNNs requires a large number of samples, but it is difficult to meet the training requirements because the acquisition of ship-radiated noise samples is very expensive. Based on the GAN model and DCGAN model, the conditional deep convolutional GAN (cDCGAN) model is designed to augment the feature samples. The expanded samples are used to train the classifier based on ResNet.

The proposed classification system is mainly composed of three parts: feature extraction, data augmentation, and classification. Figure 1 shows the structure of the classification system proposed in this paper.

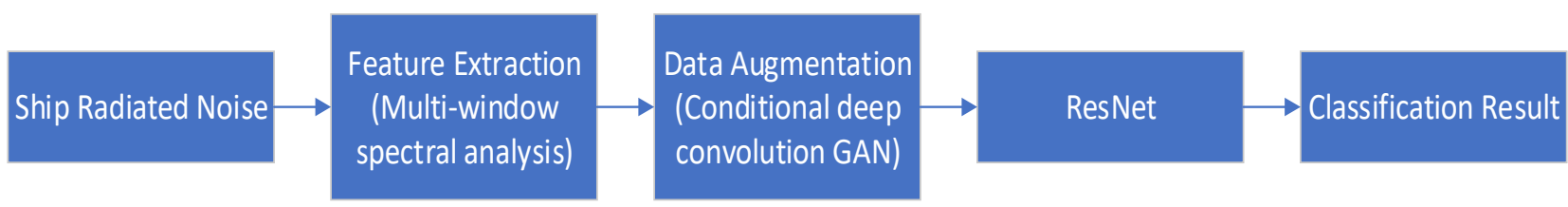

Figure 1. The structure of the proposed classification system.

In the feature extraction part, the MWSA method is used to convert the noise data into three-channel image data as the time-frequency feature of the signal, and the original sample set is constructed. In the data augmentation part, the cDCGAN model is designed to solve the problem of the insufficient number of ship-radiated noise samples. This 
method can effectively increase the number of samples and provide sufficient samples for the training of the classification network. To improve the training efficiency of the deep network, a classifier based on ResNet is designed to classify samples.

\subsection{Multi-Window Spectral Analysis}

The energy of ship-radiated noise signals is usually concentrated in a certain limited frequency band and there is a large amount of redundant information in signal waveform data. Ship-radiated noise has local stationary characteristics. T-F analysis is an effective data preprocessing method. LOFAR analysis is a common method for shipradiated noise signal analysis, which is generally implemented based on short-time Fourier transform (STFT).

For the signal $x(t)$, its STFT is defined as:

$$
\operatorname{STFT}(t, f)=\int_{-\infty}^{\infty}\left[x(\tau) w^{*}(\tau-t)\right] e^{-j 2 \pi f \tau} d \tau
$$

where $w(t)$ is the window function, which satisfies $\|w(t)\|=1$.

The discrete form of STFT is as follows:

$$
\operatorname{STFT}(n, k)=\sum_{m=-\infty}^{+\infty} x(m) w^{*}(m-n) e^{-j 2 \pi k m}
$$

where $w(n)$ is the time window in discrete. The typical LOFAR diagram obtained by STFT method is shown in Figure 2.

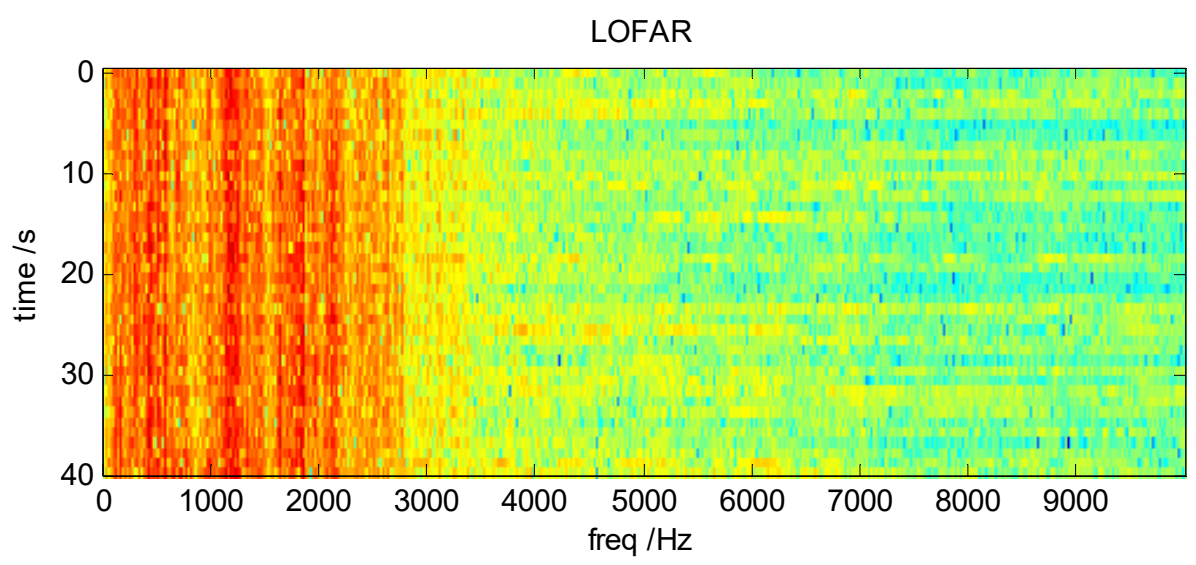

Figure 2. LOFAR of ship-radiated noise signal.

Figure 2 shows the LOFAR diagram of real ship-radiated noise signals received by SONAR processed by STFT. The classification system proposed in this paper takes the LOFAR diagram as the input of feature samples. It is worth studying how to generate LOFAR diagrams with better category properties.

The time window $w(t)$ has a decisive influence on the T-F resolution of STFT analysis. In order to obtain high resolution signal energy distribution in the joint T-F domain, it is necessary to set up an energy concentration window on the T-F plane. This energy concentration is limited by the Heisenberg-Gabo uncertainty principle, which states that for a given signal, the product of its time width and bandwidth is a constant.

It is well known that short time windows provide good time resolution, but poor frequency resolution. In contrast, long time windows provide good frequency resolution, but poor time resolution.

In order to compare the time resolution and frequency resolution of various windows, the window can be described by the parameters of "time center", "time width", "frequency center" and "frequency width". For $w(t)$, the definitions of these parameters are shown in Table 1 , where $W(j \Omega)$ is the form in frequency domain for $w(t)$. 
Table 1. The definitions of window parameters of $w(t)$.

\begin{tabular}{cc}
\hline Name & Expression \\
\hline $\begin{array}{c}\text { signal energy }(E) \\
\text { time center }\left(t_{0}\right)\end{array}$ & $E=\left.|| w(t)\right|^{2}=\int|w(t)|^{2} d t=\frac{1}{2 \pi} \int|W(j \Omega)|^{2} d \Omega$ \\
$t_{0}=\frac{1}{E} \int t|w(t)|^{2} d t$ \\
frequency center $\left(\Omega_{0}\right)$ \\
time width $\left(\Delta_{t}\right)$ \\
$\Omega_{0}=\frac{1}{2 \pi E} \int \Omega|W(j \Omega)|^{2} d \Omega$ \\
frequency width $\left(\Delta_{\Omega}\right)$ & $\Delta_{\Omega}=\left(\frac{1}{2 \pi E} \int(\Omega-\Omega)^{2}|W(j \Omega)|^{2} d \Omega=\frac{1}{2 \pi E} \int \Omega^{2}|W(j \Omega)|^{2} d \Omega-\Omega_{0}^{2}\right)^{\frac{1}{2}}$ \\
\hline
\end{tabular}

Theoretically, the Gaussian window has the smallest $\Delta_{t} \Delta_{\Omega}=0.5$ among all the windows, which means that it has the best energy aggregation performance in the T-F plane.

For finite length digital windows used in digital signal processing, it is not easy to calculate the corresponding $\Delta_{t}$ and $\Delta_{\Omega}$ analytically. For the convenience of calculation, the effective width in frequency domain and time domain is redefined here. The signal time width is defined as the time from the signal center as the symmetry center to both sides until it contains $80 \%$ energy area. Similarly, the spectrum width of a signal is defined as the spectrum width from the symmetry center of the signal to both sides until it contains $80 \%$ energy area. The rectangular, Hanning, Hamming, and Blackman windows are analyzed by numerical calculation. The results are shown in Figure 3. Although the time bandwidth product of a Gaussian window can reach the minimum in theory, the actual Gaussian window is truncated, which has an impact on the performance of the window. The results of numerical analysis of different $\alpha$-parameter Gaussian windows and truncated Gaussian windows, are shown in Figures 4 and 5. The analysis results of the above windows are shown in Table 2.
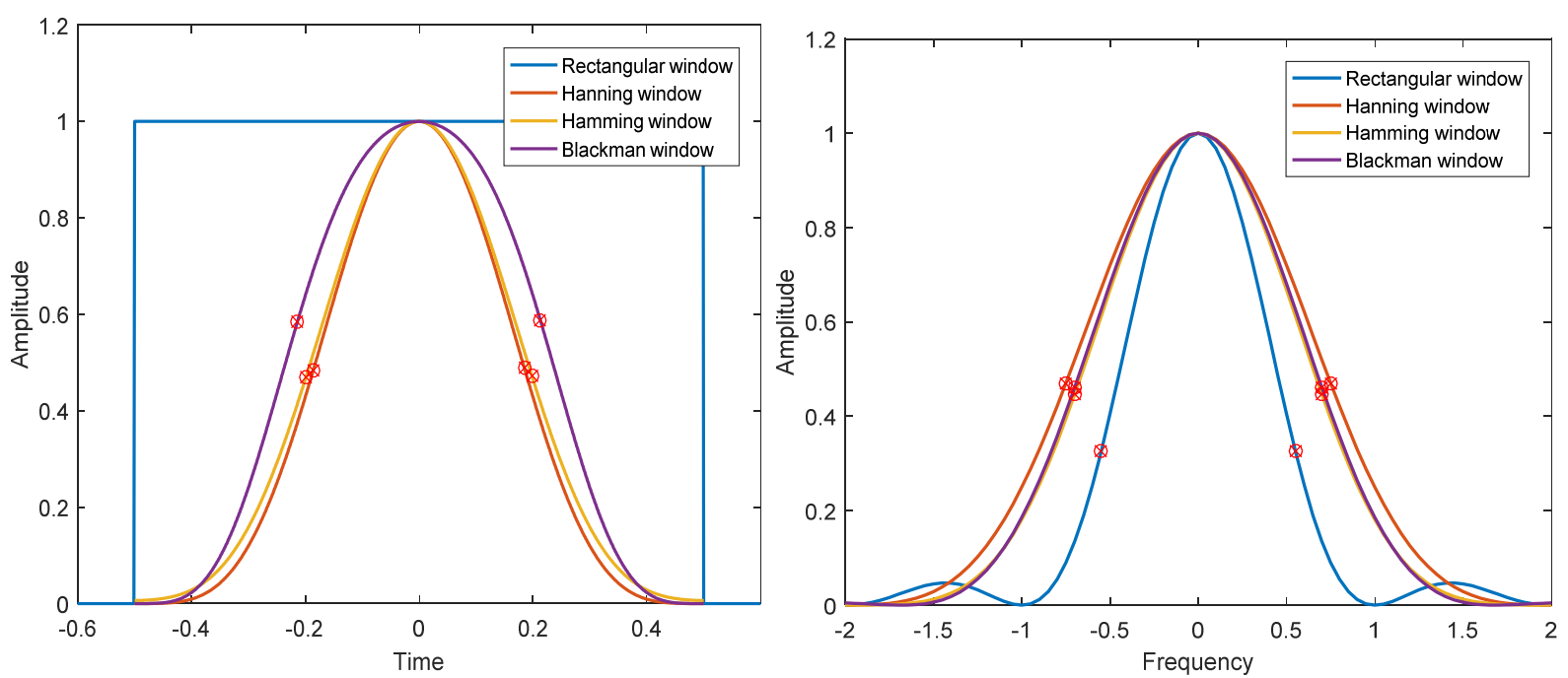

Figure 3. Time domain (left) and frequency domain (right) of different windows.

The following conclusions can be drawn from Figures 3-5 and Table 2. (1) The rectangular window has the clearest time boundary, but there is serious spectrum leakage in the frequency domain, which leads to poor frequency resolution and relatively large time bandwidth product. (2) Hanning, Hamming, and Blackman windows have similar performance. The fluctuation of the spectrum is much lower than that of the rectangular window, and the time bandwidth product is close to the Gaussian window. Among the three windows, the Hamming window has the smallest time bandwidth product. (3) The energy of the Gaussian window is the most concentrated in T-F domain, and there is no fluctuation in time domain and frequency domain. However, the truncated Gaussian window has fluctuations in the spectrum, which makes the spectrum characteristics worse and 
widens the bandwidth. As can be seen from Table 2, the time bandwidth product of the truncated Gaussian window is almost twice as large as that of the uncut Gaussian window. Through the above analysis, we can know that the Hamming window has the best energy concentration characteristics in practical application.
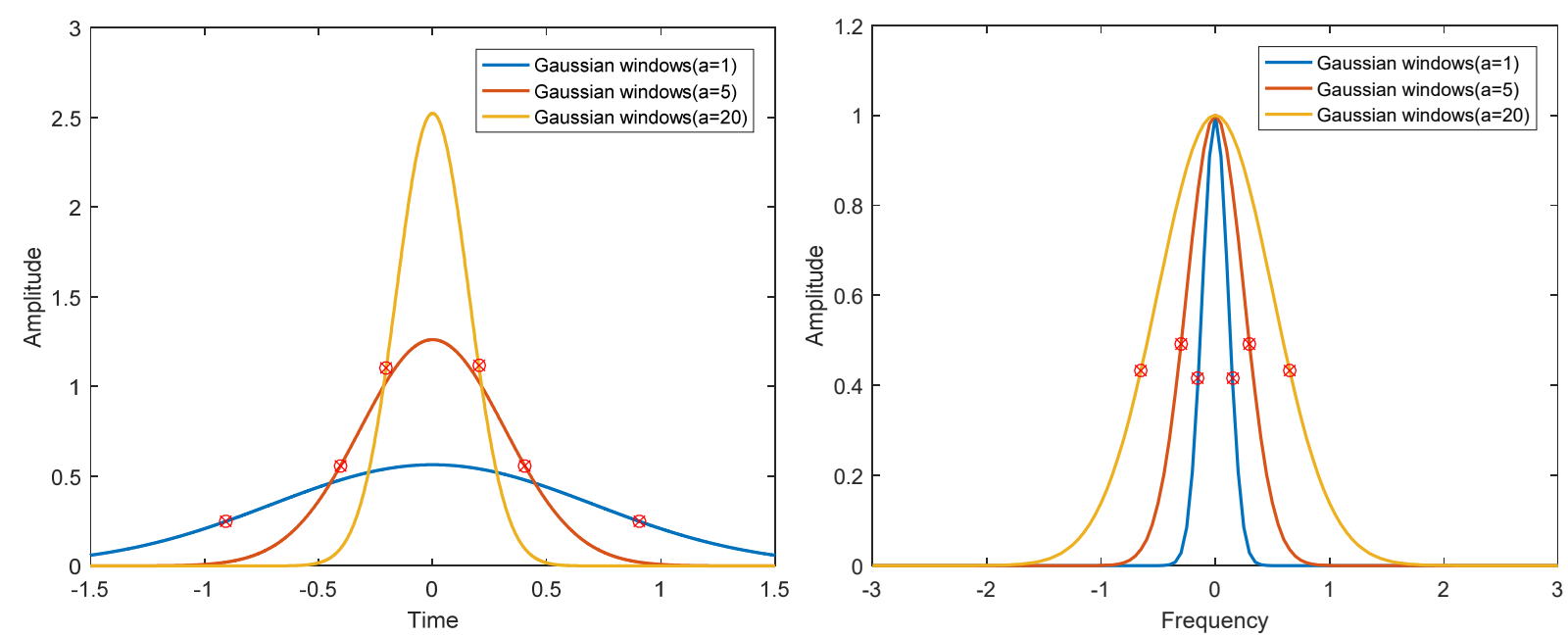

Figure 4. Time domain (left) and frequency domain (right) of different Gaussian windows.
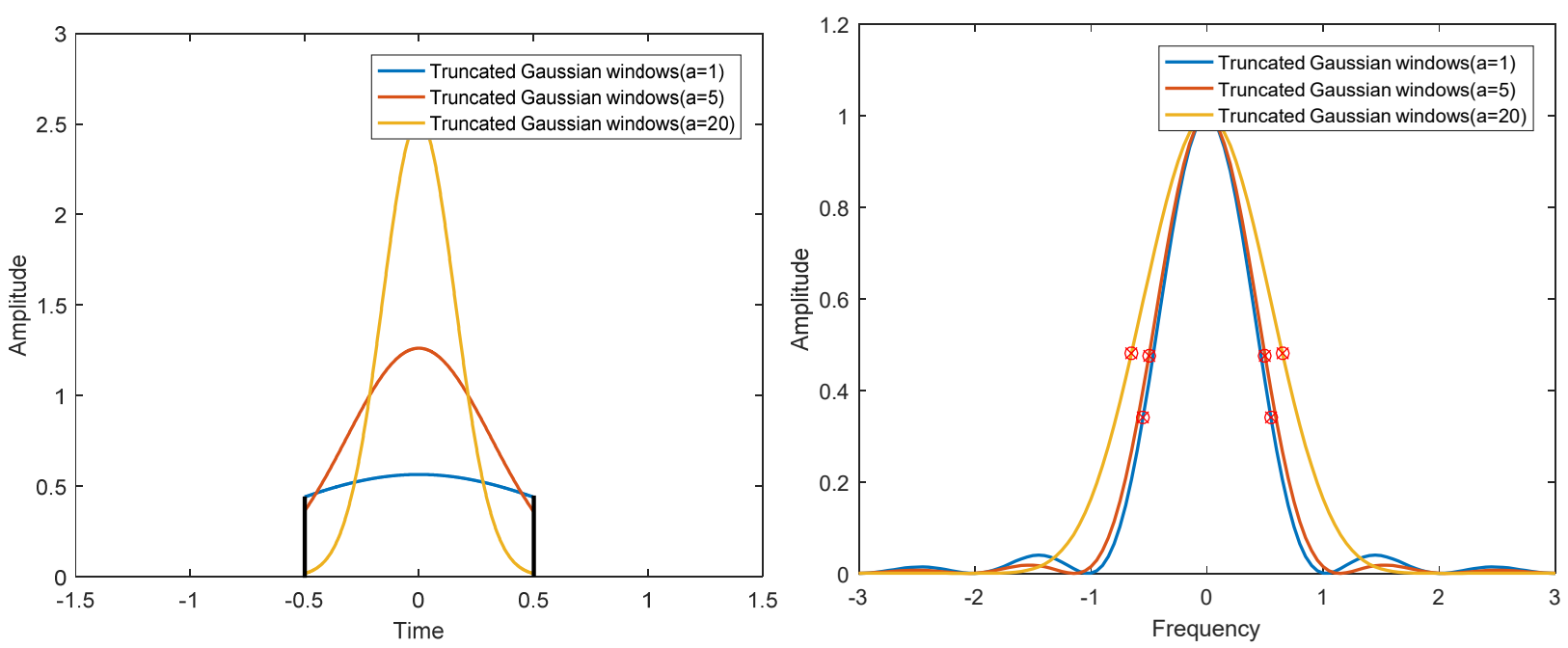

Figure 5. Time domain (left) and frequency domain (right) of different truncated Gaussian windows.

Table 2. Parameters of various windows (When $f_{s}=1 \mathrm{kHz}$ and $\mathrm{M}=1000$ ).

\begin{tabular}{cccc}
\hline Window Function & $\Delta_{t}$ & $\Delta_{\Omega}$ & $\Delta_{t} \Delta_{\Omega}$ \\
\hline Rectangular & 1 & 1.14 & 1.14 \\
Hanning & 0.38 & 1.52 & 0.578 \\
Hamming & 0.404 & 1.26 & 0.549 \\
Blackman & 0.43 & 1.42 & 0.611 \\
Gaussian $(\alpha=1)$ & 1.794 & 0.32 & 0.574 \\
Gaussian $(\alpha=5)$ & 0.812 & 0.66 & 0.536 \\
Gaussian $(\alpha=20)$ & 0.406 & 1.28 & 0.519 \\
Truncated Gaussian $(\alpha=1)$ & 1 & 1.08 & 1.08 \\
Truncated Gaussian $(\alpha=5)$ & 1 & 1.12 & 1.12 \\
Truncated Gaussian $(\alpha=20)$ & 1 & 1.3 & 1.3 \\
\hline
\end{tabular}

To improve the feature extraction capability for signals in the T-F domain, it is effective to perform T-F transformations of signals through multiple windows. A set of windows 
with different time widths applied to the signal will produce a batch of spectrums with different T-F resolutions. According to the above method, the final multi-resolution $\mathrm{M}$ graph data can be expressed by the following formula:

$$
\operatorname{MW} \_\operatorname{STFT}(n, k, i)=\alpha_{i}\left|\sum_{m=n-\frac{N}{2}}^{n+\frac{N}{2}-1} x(m) w_{i}(m-n) e^{-j 2 \pi \frac{m}{N} k}\right|^{2}
$$

where, $i$ represents the $i$-th window function and the $i$-th channel of the multi-resolution spectrograms. $\alpha_{i}$ represents the $i$-th weight. $w_{i}(n)$ represents the $i$-th window function. $N$ represents the length of the windows. In order to obtain different time-frequency resolutions and make the data obtained from multiple window functions have the same size in the time domain, this paper constructed a set of window functions based on Hamming windows, whose specific expressions are as follows:

$$
w_{i}(n)=\left\{\begin{array}{cc}
0.54-0.46 \cos \left(\frac{2 \pi\left(n-\frac{N}{2}\right)}{\frac{N}{1}-1}\right), & -\frac{N}{2} \leq n-\frac{N}{i}<\frac{N}{2} \\
0, & \text { otherwise }
\end{array}\right.
$$

In order to reduce the influence brought by the length of the window function, the weight of the window function $\alpha_{i}$ is set as follows:

$$
\alpha_{i}=\frac{1}{H}\left(\sum_{n=-\frac{N}{2}}^{\frac{N}{2}-1} h_{0}(n) / \sum_{n=-\frac{N}{2}}^{\frac{N}{2}-1} h_{i}(n)\right), i=1,2, \ldots, H
$$

The method of MWSA is illustrated by an example of simulation signal processing. The simulation signal consists of white noise and six narrowband components. The sampling rate of the signal is $32 \mathrm{kHz}$ and the duration of the signal is $30 \mathrm{~s}$. The starting and ending times of the six narrow band components $\mathrm{C} 1-\mathrm{C} 6$ are $5 \mathrm{~s}$ and $25 \mathrm{~s}$ respectively. The parameters of narrow band components are shown in Table 3.

Table 3. Parameters of simulation signal.

\begin{tabular}{cccc}
\hline & Signal Type & Frequency $\mathbf{( H z )}$ & Time (s) \\
\hline C1 & CW & 50 & $0 \sim 30$ \\
C2 & CW & 55 & $0 \sim 30$ \\
C3 & LFM & $350 \sim 50$ & $0 \sim 30$ \\
C4 & LFM & $355 \sim 55$ & $0 \sim 30$ \\
C5 & PULSE & $/$ & $20 \sim 20.031$ \\
C6 & PULSE & $/$ & $20.125 \sim 20.156$ \\
\hline
\end{tabular}

These six components are three pairs of similar components used to observe the performance of different windows for different types of signals. Three windows wn1-wn3 are used to implement MWSA. The Hamming window is used in all three windows, and the corresponding time lengths are $0.125 \mathrm{~s}, 0.5 \mathrm{~s}$, and $2 \mathrm{~s}$. The time domain and frequency domain shapes of each window are shown in Figure 6.

As can be seen from Figure 6, wn1 has the highest time resolution and the lowest frequency resolution in all windows. C5/C6 can clearly be distinguished in the LOFAR obtained by wn1, but it is difficult to distinguish $\mathrm{C} 1 / \mathrm{C} 2$ and $\mathrm{C} 3 / \mathrm{C} 4$. It is difficult to distinguish the $\mathrm{C} 1 / \mathrm{C} 2$ signals in the LOFAR obtained by wn2, but it has good resolution for $\mathrm{C} 3 / \mathrm{C} 4$ and $\mathrm{C} 5 / \mathrm{C} 6$. The time resolution of $\mathrm{wn} 3$ is low and it is difficult to distinguish the adjacent pulse. However, wn3 has the best frequency resolution. 


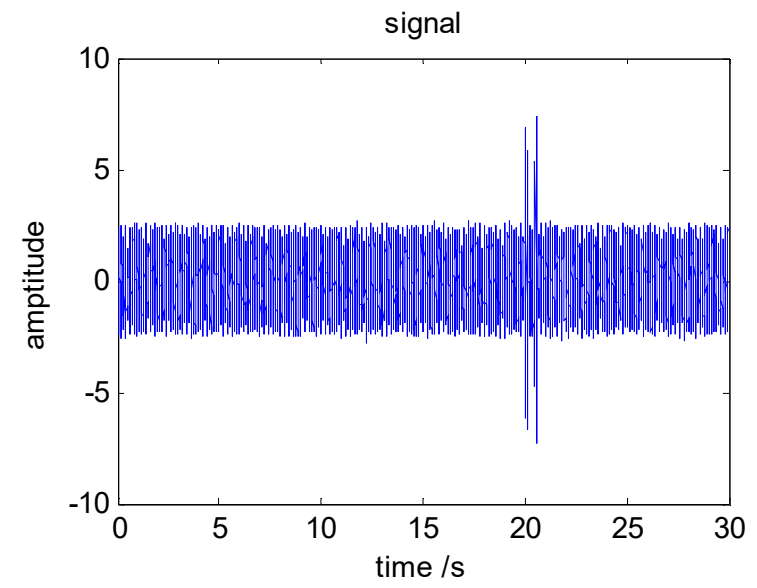

(a)

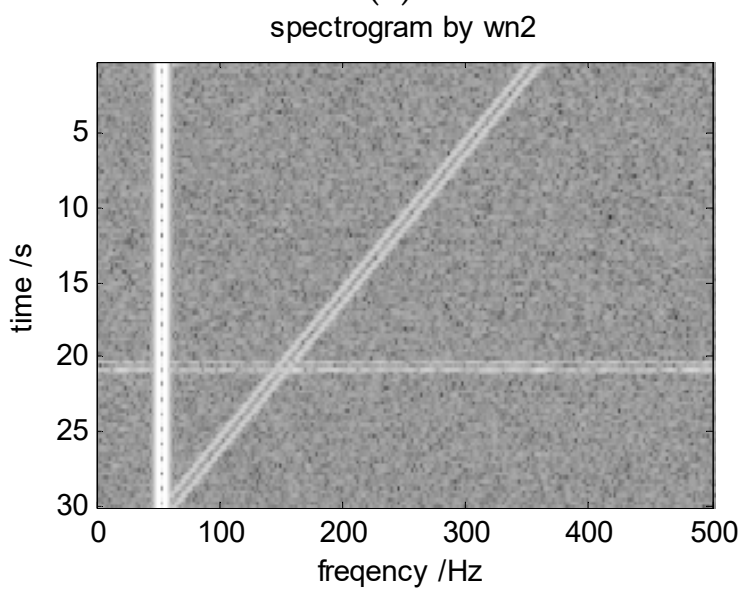

(c)

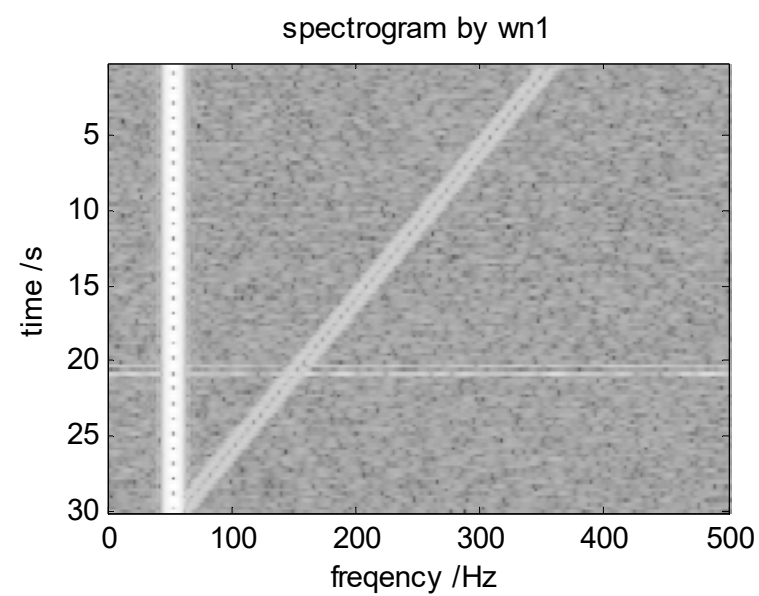

(b)

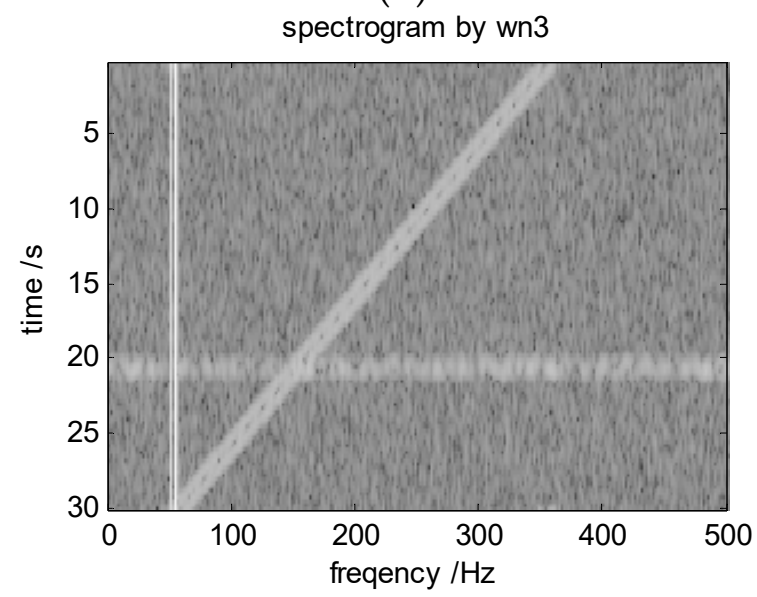

(d)

Figure 6. Signal waveform (a) and spectrograms of the signal (b) by wn1, (c) by wn2, and (d) by wn3.

The duration of ship-radiated noise signals is not fixed. For uniformity of sample set data, the ship-radiated noise signals should be divided into several frames of fixed length through a window of suitable width. According to the MWSA method in Section 2, three window functions are set to process the sample data, and the three obtained spectrograms are stored in three channels of a color image to form the final sample. Figure 7 shows a schematic diagram of a sample construction.
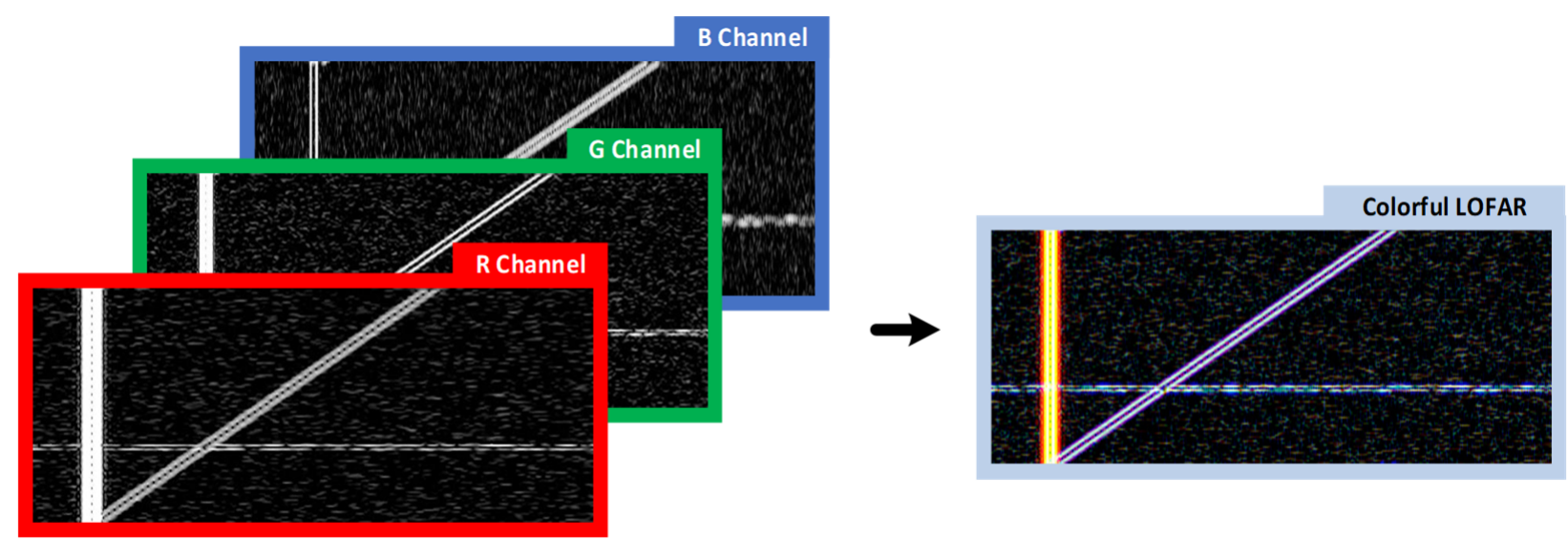

Figure 7. Sample storage method for multi-resolution spectrum analysis. 


\subsection{Conditional Deep Convolutional GAN Model}

Due to the high cost of acquiring ship-radiated noise, it is difficult to obtain sufficient samples to support the training of the classifier. In this paper, the cDCGAN model is designed based on the GAN model to expand the number of samples.

GAN consists of a generator $(G)$ and a discriminator $(D)$. The purpose of the generating model is to make the new generated sample as similar as possible to the training sample, while the purpose of the discriminator model is to distinguish the real sample from the generated sample as accurately as possible.

The original GAN model has two shortcomings: (1) the model does not contain label information, so the training efficiency is low; and (2) the connection structure of the generator and discriminator is simple, and the generation ability for complex samples is weak. For the problem of label information, Ref. [22] proposed the CGAN model, which introduced label information into the training process. To improve the performance of the generator and discriminator, the DCGAN model was proposed in Ref. [23], and the structure of a convolutional neural network was introduced into a GAN, which achieved good results. The cDCGAN model proposed in this paper integrates the above two models and improves them. Figure 8 shows the structure of the cDCGAN.

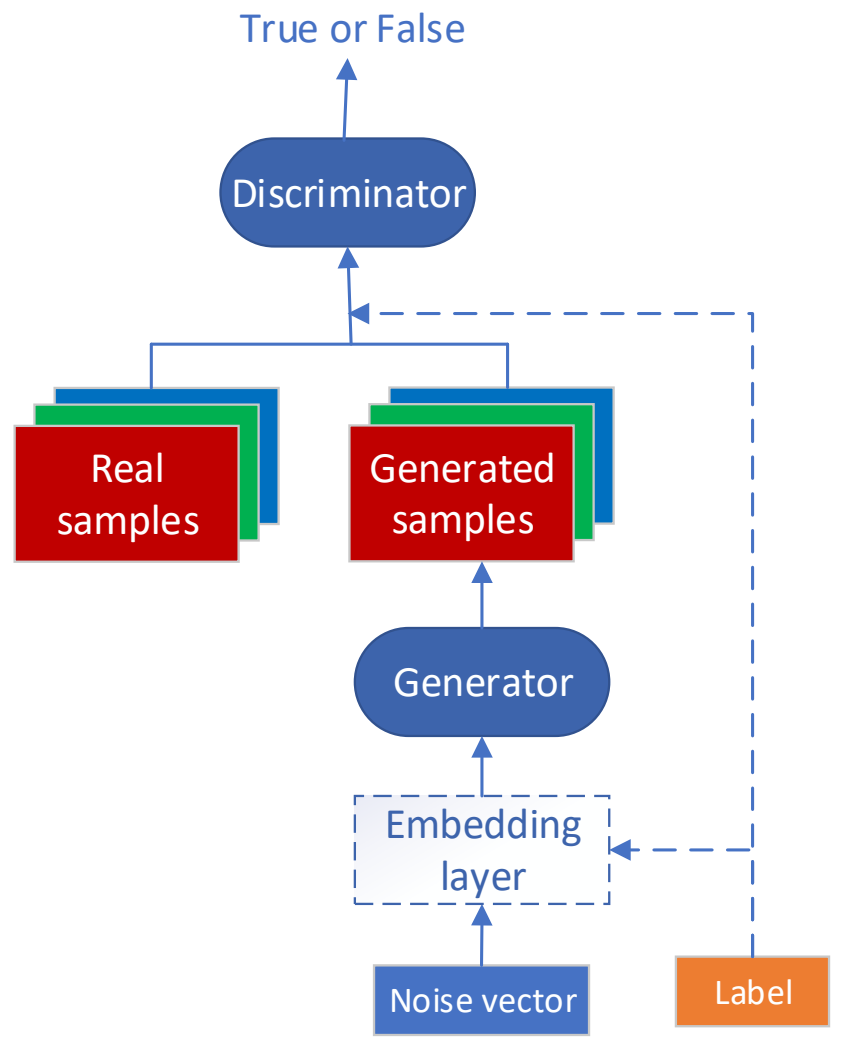

Figure 8. Structure of the cDCGAN.

In the CGAN, the input vector is composed of a label vector and a random noise vector. The dimension extension of the generator input vector increases the processing complexity. As shown in Figure 8, the cDCGAN model is improved based on CGAN by introducing an embedding layer into the generator model. In the embedding layer, the label vector is converted to a vector of the same size as the noise vector, and then the two vectors are multiplied by elements to fuse the label information into the input noise without changing the size of the input vector. The embedding layer transforms sparse noise vectors and label vectors into dense input vectors. In the cDCGAN, the label of the sample is entered into the generator to make the generated sample category consistent with the input label. 
The discriminator not only determines whether the input sample is real, but also needs to judge whether the output label is correct.

Similar to the DCGAN, the CDCGAN model improves the generator and discriminator of the GAN model; the structure of the improved generator and discriminator is shown in Figures 9 and 10.

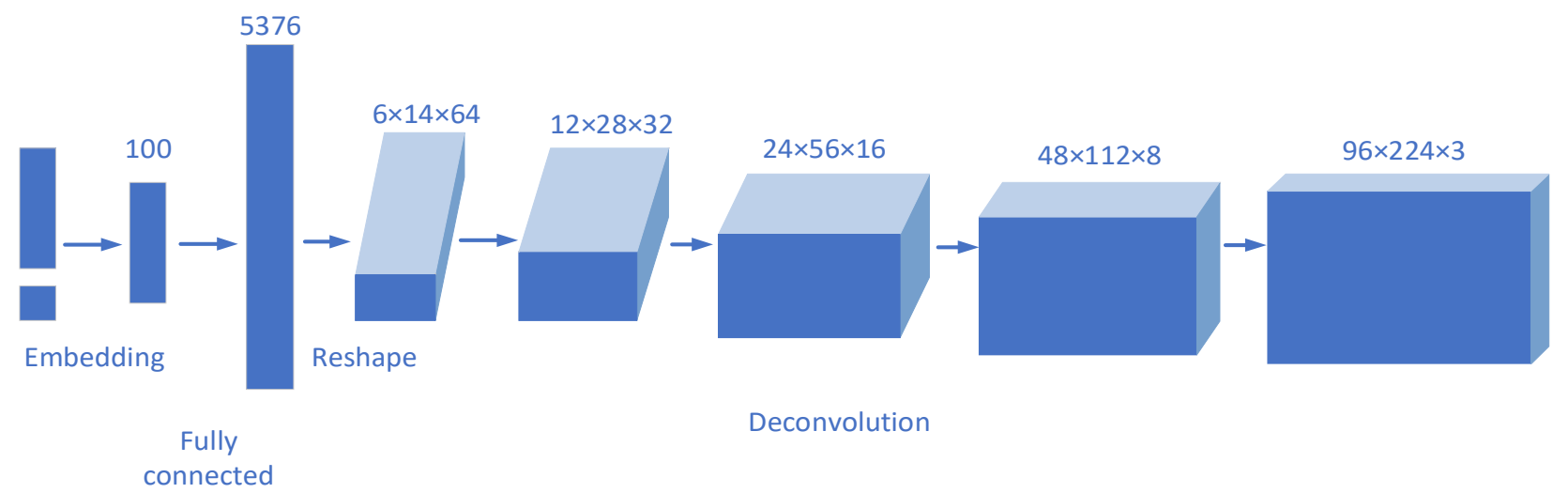

Figure 9. Generator structure.

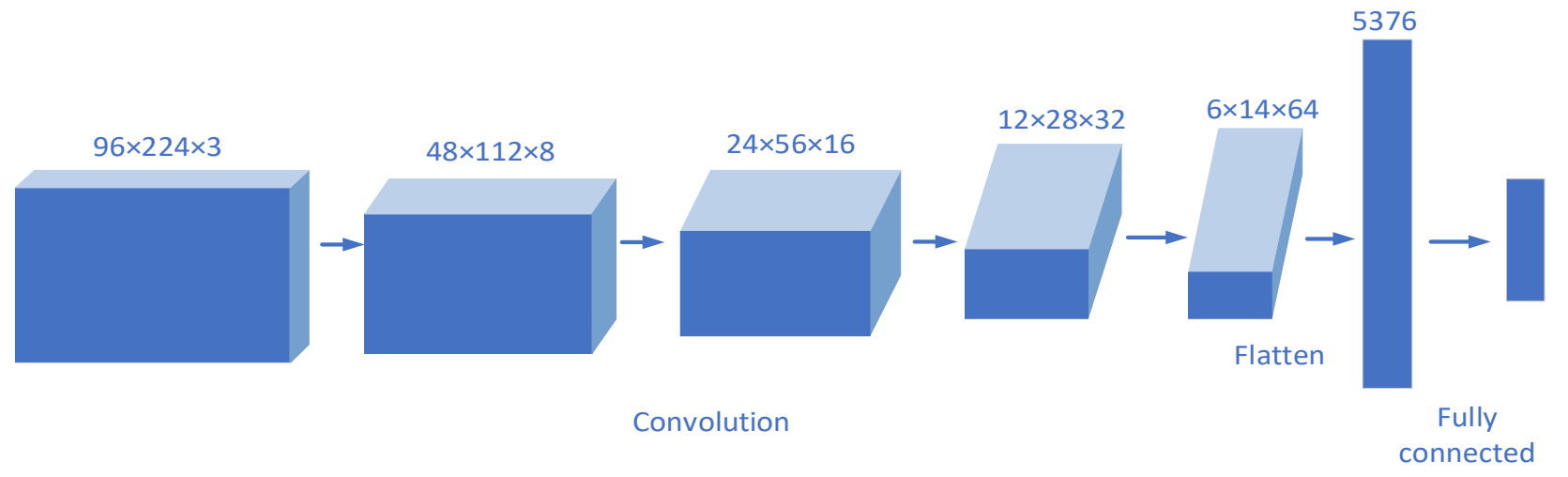

Figure 10. Discriminator structure.

In the generator, the input vector is a fusion of random normally distributed noise and label information. First, the data size is enlarged through the fully connected layer, and then the data are reconstructed to transform the one-dimensional data into threedimensional data of $6 \times 14 \times 64$. Then, up-sampling is carried out step by step through the deconvolution layer and a convolution kernel with a size of $5 \times 5$ and a step size of 2 is used in each layer. In each layer, the size of the feature graph is doubled, and finally, after four deconvolutions, the image is gradually amplified into a 3-D image of $96 \times 224 \times 3$.

In the discriminator, the real samples are mixed with the generated samples as the data set. The characteristics of input samples are gradually learned by down-sampling through multiple convolution layers. A convolution kernel with a size of $5 \times 5$ and a step size of 2 is used in each layer, and the size of the feature graph is reduced by half in each layer. After four convolution layers, the 3-D data of $6 \times 14 \times 64$ are flattened into 1-D data and then inputted into a fully connected layer. Finally, the Sigmoid function and the SoftMax function are used to obtain the authenticity and category of the sample. The judgment of the discriminator is considered correct only when the authenticity and category of samples are both correct.

The training of the GAN is the process of game confrontation between the generator and the discriminator. The purpose of the generator is to maximize the probability of incorrect judgment of the discriminator, while the purpose of the discriminator is to 
maximize the probability of correct judgment. With the addition of label information, the loss function in the cDCGAN can be expressed as follows:

$$
\min _{G} \max _{D} V(D, G)=E_{x \sim p_{x}(x)}[\log D(x \mid c)]+E_{z \sim p_{z}(z)}[\log (1-D(x \mid c))]
$$

where $V(D, G)$ is the value function of cDCGAN. $D$ is the output of the discriminator. $G$ is the output of the generator. $p_{x}(x)$ is the distribution of real samples and $p_{z}(z)$ is the distribution of random noises.

\subsection{Classifier Based on ResNet}

In deep networks, generally, the network accuracy should increase as the network depth increases. However, as the network gets deeper, a new problem arises. These layers bring a large number of parameters that need to be updated. When the gradient propagates from back to front, the gradient of the earlier layer will be very small when the network depth increases. This means that the learning of these layers stagnates, which is the vanishing gradient problem. In addition, more neural network layers mean that the parameter space is larger and parameter optimization becomes more difficult. Simply increasing the network depth will lead to higher training errors. This is not because of overfitting (the training error of the training set is still very high), but because of network degradation. ResNet designs a residual module that can effectively train deeper networks.

For the deep network structure, when the input is $x$, the learned features are denoted as $H(x)$, and the target learning feature can be changed to $F(x)=H(x)-x$. The reason for this is that residual learning is easier than raw feature learning. When the residual is 0 , the accumulation layer only does the identity mapping and, at least, the network performance does not decline. In fact, the residual will not be 0 , which will also enable the accumulation layer to learn new features based on the input features, so as to have better performance. This is similar to a "short circuit" in a circuit, so it is called a shortcut connection. The structure of residual learning is shown in Figure 11 [19].

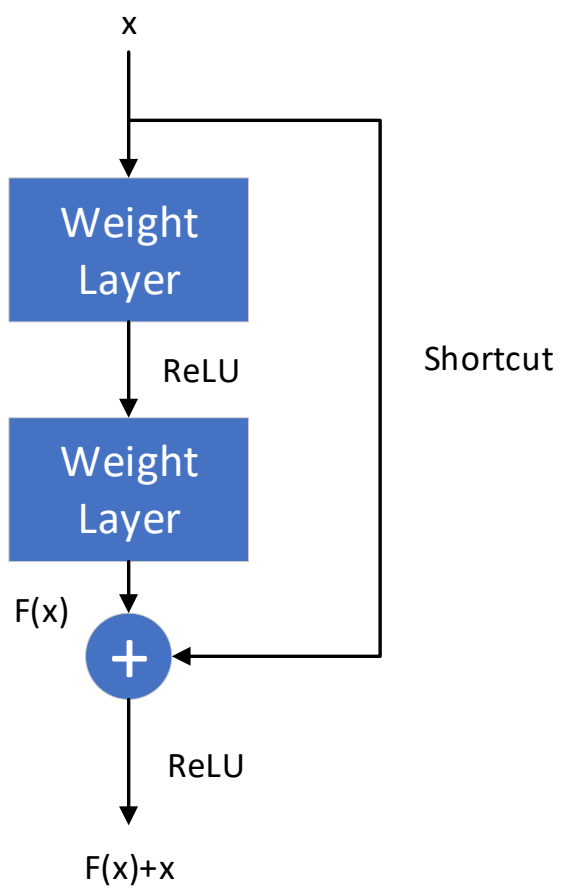

Figure 11. Residual module structure.

The residual module can be expressed as:

$$
x_{l+1}=f\left(h\left(x_{l}\right)+F\left(x_{l}, w_{l}\right)\right)
$$


where $x_{l}$ and $x_{l+1}$ represent the input and output of the $l$-th residual unit, respectively, and each residual module generally contains a multi-layer structure. $w_{l}$ represents the weight from the $l$-th residual unit to the $(l+1)$-th residual unit. $F$ is the residual function, representing the learned residual, while $h\left(x_{l}\right)=x_{l}$ represents the identity mapping, and $f$ is the ReLU activation function. Based on the above formula, learning characteristics from shallow $l$ to deep $L$ can be obtained:

$$
x_{L}=x_{l}+\sum_{i=l}^{L-1} F\left(x_{i}, w_{i}\right)
$$

Using the chain rule, the gradient of back propagation can be roughly obtained:

$$
\frac{\partial \text { loss }}{\partial x_{l}}=\frac{\partial \text { loss }}{\partial x_{L}} \cdot \frac{\partial x_{L}}{\partial x_{l}}=\frac{\partial \text { loss }}{\partial x_{L}} \cdot\left(1+\frac{\partial}{\partial x} \sum_{i=l}^{L-1} F\left(x_{i}, w_{i}\right)\right)
$$

In ResNet, the residual module mainly has two forms, one is the identity block that keeps dimension unchanged, the other is the convolution block that changes dimension. Figure 12 shows the main structures of the two blocks.
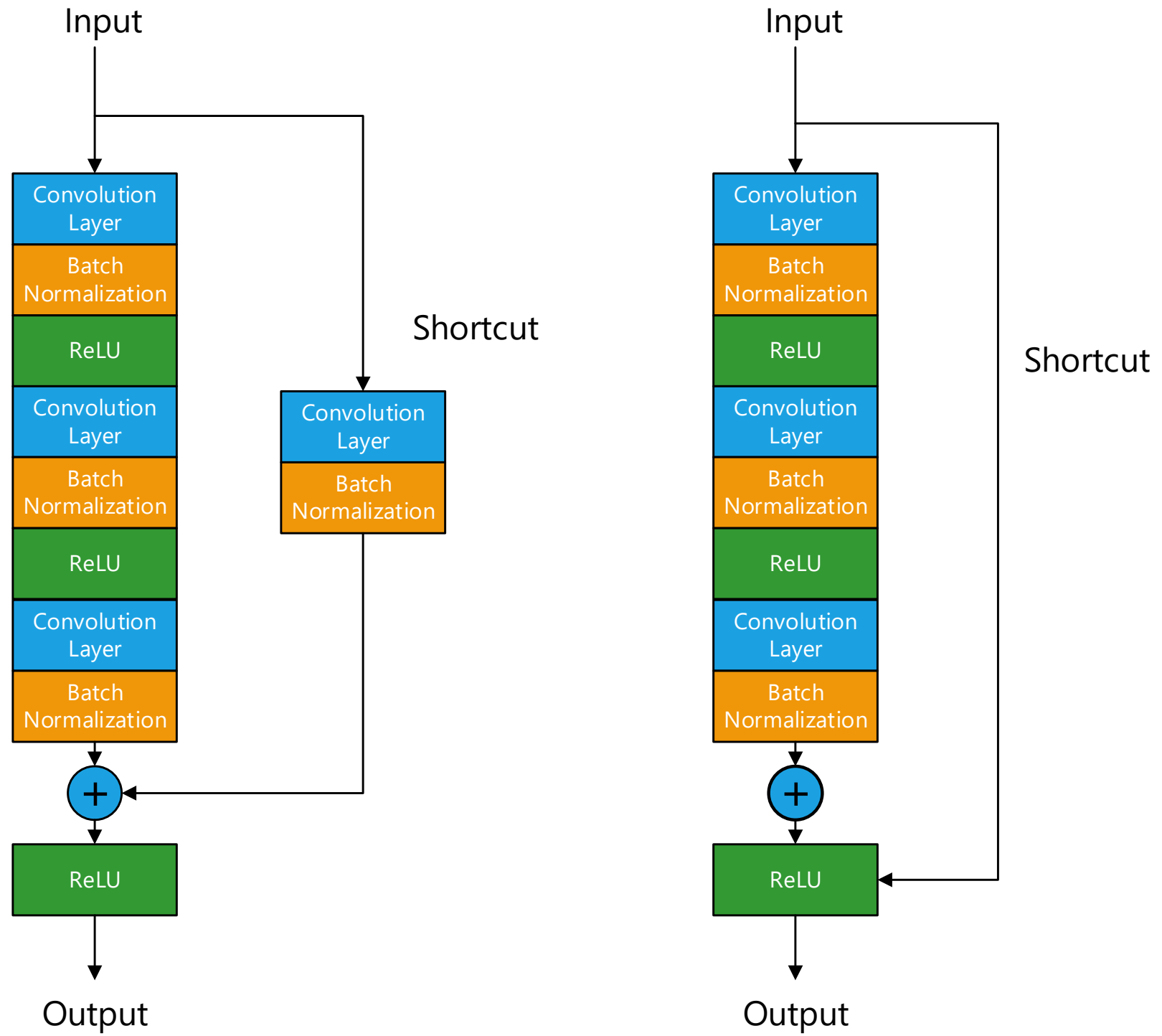

Figure 12. Convolution block and identity block. 
This paper designed a classification network based on ResNet; its structure is shown in Figure 13.
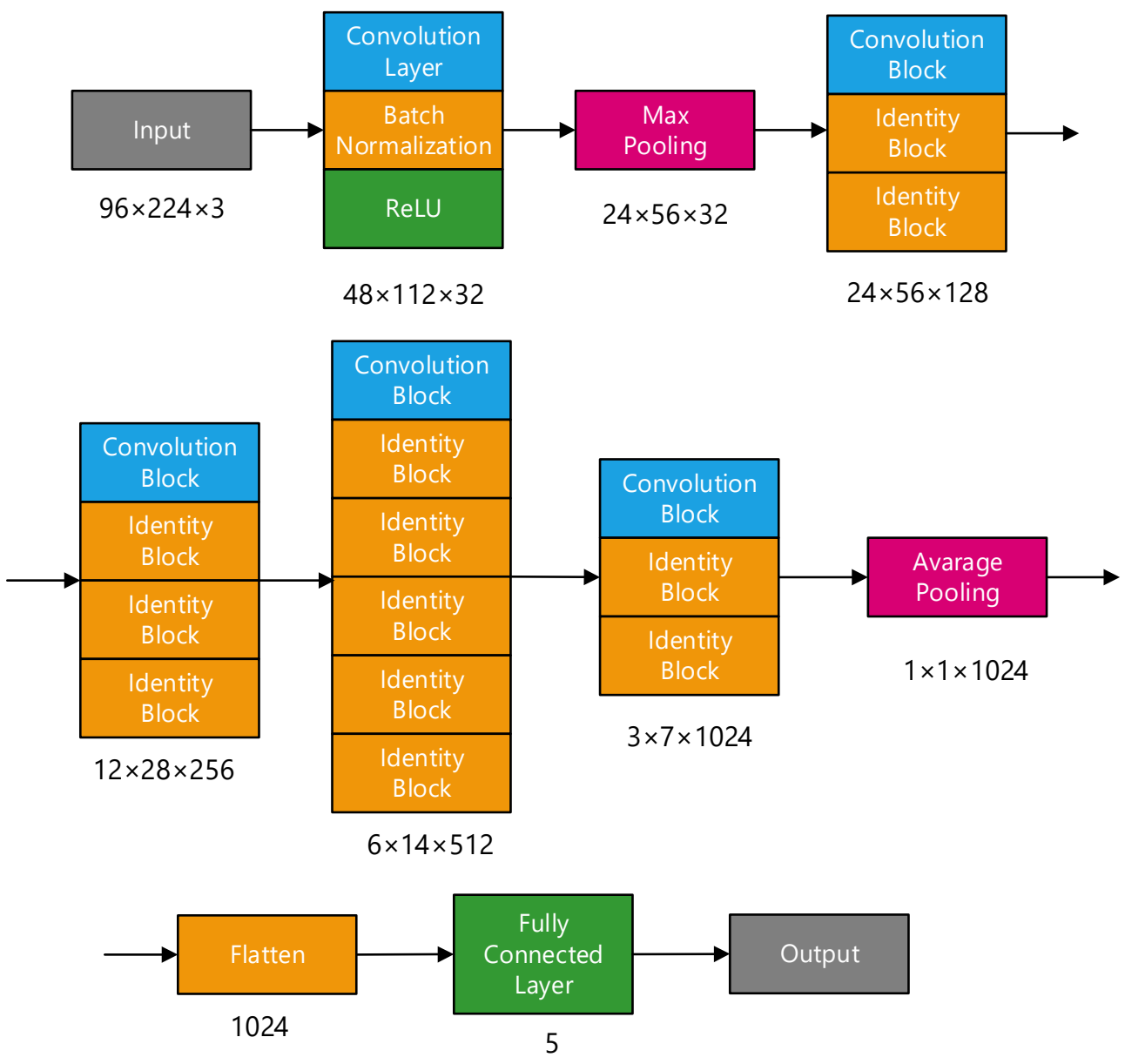

Figure 13. ResNet structure.

After feature extraction, the input sample size was $96 \times 224 \times 3$. First, the convolution layer, batch normalization, and ReLU activation function were used to change the size to $48 \times 12 \times 32$ by using 32 convolution kernels, and then the size was further reduced by maximum pooling. The core part of the network is composed of multiple convolution modules and identity modules. Each convolution module will halve in size and adopt a residual structure. Even if the network deepens, the problem of vanishing gradient and exploding gradient can be effectively solved. Finally, after average pooling, the size is changed to $1 \times 1 \times 1024$. After flattening to one-dimensional data, the category of the network is output through the full connection layer. In the network, the size of the convolution kernels is $3 \times 3$, and the step size is 2 . Meanwhile, to ensure that the total amount of learnable parameters remains unchanged, the number of convolution kernels will double every time the size of the feature graph is halved.

\section{Experiment and Analysis}

\subsection{Experimental Database}

To test the performance of the proposed UATR system, a test sample set was constructed based on the ShipsEar database [24]. The ShipsEar database is composed of 90 records representing sounds from 11 vessel types. It includes detailed information on technical aspects of the recordings and environmental and other conditions during acquisition. These recordings include many different types of vessels from the docks, including fishing boats, ocean liners, ferries of various sizes, container, roros, tugs, pilot boats, yachts, small sail boats, etc. The recordings were made with autonomous acoustic 
digitalHyd SR-1 recorders. This compact recorder includes a hydrophone with a nominal sensitivity of $-193.5 \mathrm{~dB}$ re $1 \mathrm{~V} / 1 \mathrm{uPa}$ and a flat response in the $1 \mathrm{~Hz}-28 \mathrm{kHz}$ frequency range. The amplifier chain consists of a preamplifier with a high-pass cutoff frequency of $100 \mathrm{~Hz}$. The device also includes a 24-bit A/D sigma-delta converter with a sampling rate of 52,734 Hz. During data acquisition, the hydrophones were bottom-moored and attached to a submerged buoy. The distances between the recorder and the ships are less than $100 \mathrm{~m}$, and some distances are less than $50 \mathrm{~m}$. These distances ensure that a single hydrophone can receive ship-radiated noise with a high SNR.

Based on ship size, 11 ship types were reclassified into five categories, as shown in Table 4.

Table 4. ShipsEar data classification.

\begin{tabular}{cc}
\hline Category & Type of Vessel \\
\hline Class A & fishing boats, trawlers, mussel boats, tugboats and dredgers \\
Class B & motorboats, pilot boats and sailboats \\
Class C & passenger, ferries \\
Class D & ocean liners and ro-ro vessels \\
Class E & background noise recordings \\
\hline
\end{tabular}

\subsection{The Construction and Augmentation of Sample Set}

The samples in the ShipsEar database are single-channel audio signals with sampling rates of $52,734 \mathrm{~Hz}$. In this paper, all data are divided into frames of $2 \mathrm{~s}$. To obtain more samples, a $50 \%$ overlap between frames is adopted. For each data frame, the time-frequency graph is obtained by using the multi-resolution spectral analysis method. To obtain information under different time resolutions and frequency resolutions, the window lengths of the three window functions are set to $20 \mathrm{~ms}, 80 \mathrm{~ms}$, and $320 \mathrm{~ms}$ respectively, and the frequency range to $0-3000 \mathrm{~Hz}$. Subsequently, the obtained spectrograms are normalized to zero mean and unit variance and then intercepted to three standard deviations [26]. Finally, three spectrograms are stored in three channels respectively to form the final sample with a dimension of $96 \times 224 \times 3$. Figure 14 shows the spectrogram obtained by each window and the integrated spectrogram in color.

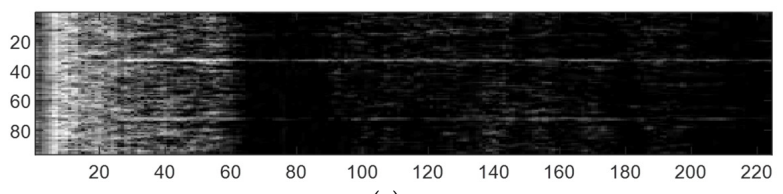

(a)

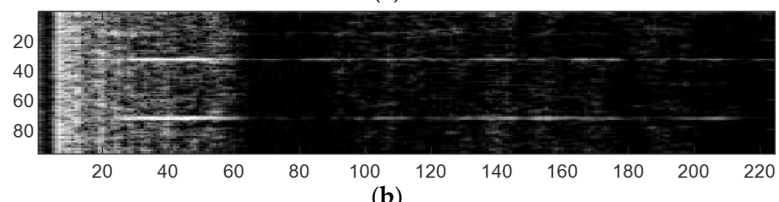

(b)

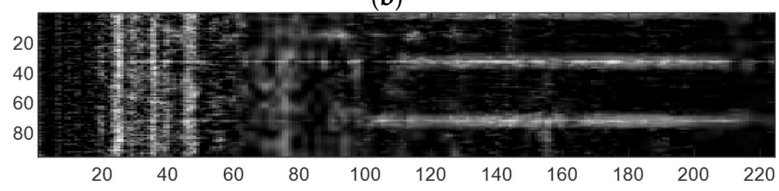

(c)

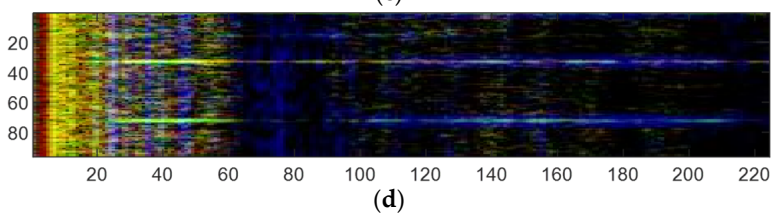

Figure 14. (a) Spectrogram by $20 \mathrm{~ms}$ window, (b) spectrogram by $80 \mathrm{~ms}$ window, (c) spectrogram by $320 \mathrm{~ms}$ window, (d) spectrogram by MWSA. 


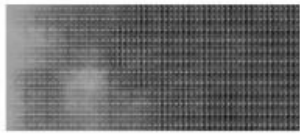

10 iterations

As can be seen from Figure 14, spectrograms of different resolutions can be saved in a single-color spectrogram by MWSA processing. In this way, the spectrogram can be easily processed by the general image classification network. These colored spectrograms form an original set of samples for classification.

The cDCGAN model is trained by the original sample set, and the corresponding generator is obtained, which can generate samples of different categories based on input labels. Figure 15 shows the sample generated by the generator after different iterations.

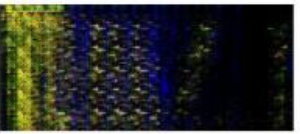

100 iterations

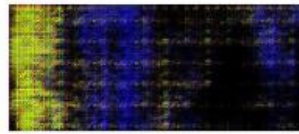

500 iterations

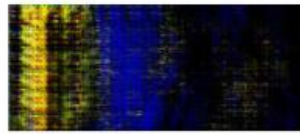

1000 iterations 2000 iterations

Figure 15. Generated samples under different iterations.

Through the DCDGAN obtained after training, we expanded the number of samples of each category in the sample base. The number of samples before and after data augmentation is shown in Table 5.

Table 5. Sample numbers before and after data augmentation.

\begin{tabular}{ccccccc}
\hline Category & A & B & C & D & E & Total \\
\hline Original number & 767 & 624 & 1690 & 1008 & 465 & 4554 \\
Expanded number & 1500 & 1500 & 2000 & 1700 & 1000 & 7700 \\
\hline
\end{tabular}

\subsection{Experimental Results}

Through the cDCGAN, various types of expanded samples are generated, and the expanded samples are combined with the original training samples to complete the data augmentation of the sample set. The enhanced training set is sent to ResNet for training, and, finally, a practical classification network can be obtained.

With the cDCGAN, the original sample set is extended. The training set was constructed by selecting samples from the extended sample set. ResNet can be trained through a training set to obtain a practical classification network.

The test set is sent to the trained ResNet network to obtain the classification results of each sample. The proposed ResNet has 50 layers. The initial learning rate is set to 0.001 , the batch size is set to 128 , and the activation function is ReLU. The confusion matrix corresponding to the classification results is shown in Table 6.

Table 6. Confusion matrix symbol representation.

\begin{tabular}{cccccc}
\hline \multirow{2}{*}{ Truth } & \multicolumn{5}{c}{ Prediction } \\
\cline { 2 - 6 } & Class A & Class B & Class C & Class D & Class E \\
\hline Class A & 183 & 2 & 5 & 2 & 0 \\
Class B & 3 & 145 & 8 & 1 & 0 \\
Class C & 4 & 3 & 409 & 5 & 2 \\
Class D & 2 & 1 & 4 & 246 & 0 \\
Class E & 0 & 0 & 0 & 0 & 117 \\
\hline
\end{tabular}

Furthermore, we use accuracy, recall rate, and F1 value as performance indicators to describe the performance of the classifier. For category $k, k=A, B, C, D, E$, each performance indicator is calculated as follows:

$$
\text { Accuracy }=\frac{n_{A A}+n_{B B}+n_{C C}+n_{D D}+n_{E E}}{N}
$$




$$
\begin{gathered}
\text { Recall }_{k}=\frac{n_{k k}}{n_{k A}+n_{k B}+n_{k C}+n_{k D}+n_{k E}} \\
\text { Precision }_{k}=\frac{n_{k k}}{n_{A k}+n_{B k}+n_{C k}+n_{D k}+n_{E k}} \\
\text { F1score }_{k}=2 \cdot \frac{\text { Precision }_{k} \cdot \text { Recall }_{k}}{\text { Precision }_{k}+\text { Recall }_{k}}
\end{gathered}
$$

where, $N$ represents the total number of test samples. $n_{i j}$ represents the number of samples of class $i$ classified as class $j$.

The accuracy of the experiment is $96.32 \%$. The values for recall, precision, and F1 score of each category are shown in Table 7.

Table 7. Experimental results.

\begin{tabular}{cccc}
\hline & Recall & Precision & F1 Score \\
\hline Class A & $95.31 \%$ & $95.31 \%$ & 0.9531 \\
Class B & $92.36 \%$ & $96.03 \%$ & 0.9416 \\
Class C & $96.69 \%$ & $96.01 \%$ & 0.9635 \\
Class D & $97.23 \%$ & $96.85 \%$ & 0.9704 \\
Class E & $100.00 \%$ & $98.32 \%$ & 0.9915 \\
Average & $96.31 \%$ & $96.50 \%$ & 0.9640 \\
\hline
\end{tabular}

As can be seen from Table 7, the lowest recall, precision, and F1 score are $92.36 \%$, $95.31 \%$, and 0.9416 , respectively. The average recall, precision, and F1 score are $96.31 \%$, $96.50 \%$, and 0.9640 , respectively. The experiment results show that the proposed UATR has good recognition ability for five categories of signals.

\subsubsection{Comparison of Feature Extraction Methods}

To test the effect of the multi-window spectral analysis method, single-channel LOFAR spectrum analysis, pseudo-color image analysis, and multi-window spectral analysis were used to extract features from ship data.

Single-channel LOFAR spectrum analysis is a general LOFAR spectrum analysis. For each data frame, the STFT is carried out, the window length is $80 \mathrm{~ms}$, the step is $20 \mathrm{~ms}$, the frequency range is $0-3000 \mathrm{~Hz}$, and the size of the final sample graph is $96 \times 224 \times 1$.

Pseudo-color image analysis is based on single-channel analysis to convert a singlechannel LOFAR spectrum into a three-channel RGB image [27], using gray value as the entrance address for the color lookup table to find the corresponding value of the three channels and then produce the color image. The purpose of pseudo-color image analysis is to improve the identifiability of the graph, but it can only provide information processed by one window function.

Figure 16 shows the sample graphs of the same data frame obtained under three different pretreatment methods. GAN data augmentation was carried out on these sample sets, and classification and identification were carried out based on ResNet. The accuracy of the test sets is shown in Table 8 .

As Table 8, compared with single-channel spectrograms, both pseudo-color spectrograms and multi-window spectrograms can effectively improve classification accuracy. Multi-window spectrograms can provide information under different T-F resolutions and have the best classification performance.

\subsubsection{Comparison of Data Augmentation Methods}

To compare the performance of the augmentation methods, six experiments were carried out based on the method of no augmentation, audio data augmentation [28], GAN augmentation, CGAN augmentation, DCGAN augmentation, and cDCGAN augmentation, respectively. The audio data augmentation method included four augmentation 
methods: time stretching (TS), pitch shifting (PS1), dynamic range compression (DRC), and background noise $(\mathrm{BG})$.

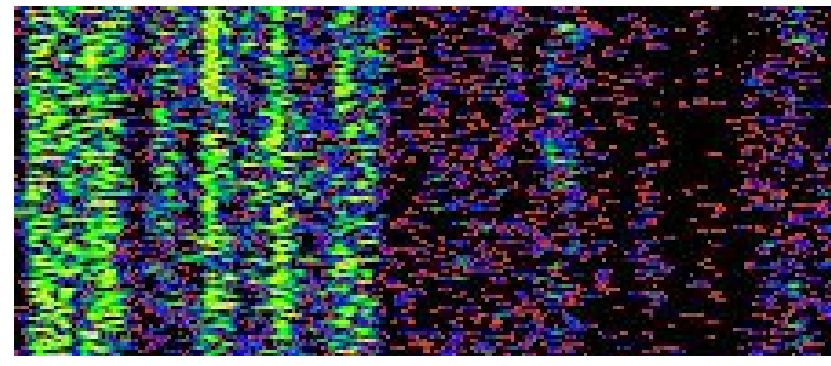

(b)

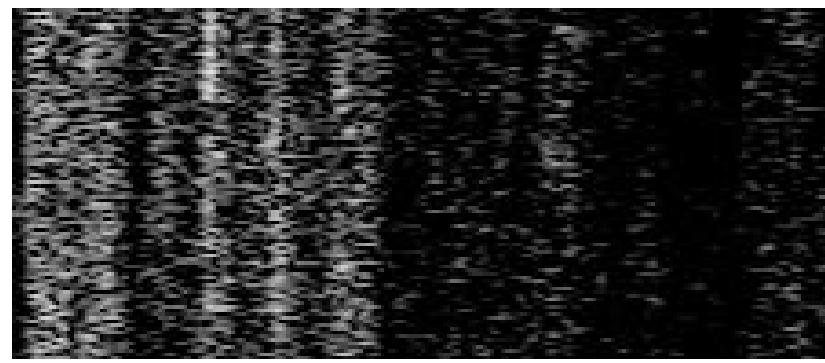

(a)

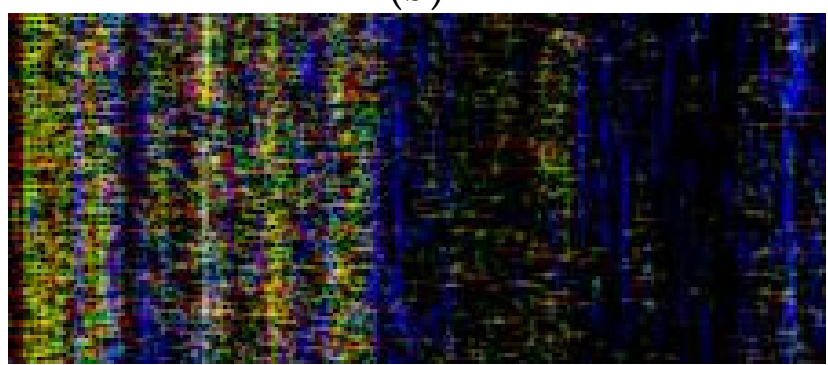

(c)

Figure 16. Samples of different preprocessing methods: (a) single-channel LOFAR spectrum $(96 \times 224 \times 1)$, (b) pseudo-color LOFAR spectrum $(96 \times 224 \times 3)$, (c) multi-resolution time-frequency spectrum $(96 \times 224 \times 3)$.

Table 8. Accuracy of different feature extraction methods.

\begin{tabular}{cc}
\hline Preprocessing Method & Accuracy \\
\hline Single-channel spectrum analysis & $92.43 \%$ \\
Pseudo-color image analysis & $93.48 \%$ \\
Multi-resolution spectrum analysis & $96.32 \%$ \\
\hline
\end{tabular}

The six experiments were trained and classified based on ResNet after multi-resolution spectrum analysis. Table 9 shows the test results of the six data augmentation methods.

Table 9. Accuracy of different data augmentation methods.

\begin{tabular}{cc}
\hline Data Augmentation Method & Accuracy \\
\hline No data augmentation & $90.94 \%$ \\
Audio data augmentation [23] & $92.60 \%$ \\
GAN data augmentation & $91.35 \%$ \\
CGAN data augmentation & $91.82 \%$ \\
DCGAN data augmentaion & $96.14 \%$ \\
cDCGAN data augmentation & $96.32 \%$ \\
\hline
\end{tabular}

It can be seen from Table 9 that data augmentation brings gains to the classification network training. Data augmentation based on cDCGAN achieves the best results.

\subsubsection{Comparison of Classification Models}

We compared the performance of the ResNet model with that of the other two classifiers. One classifier is the GMM classifier based on Ref. [24], and the other is the mature classification network VGG-19. All three experiments were based on the same training and test data sets. The test results are shown in Table 10. 
Table 10. Accuracy of different classification models.

\begin{tabular}{cc}
\hline Classification Model & Accuracy \\
\hline GMM & $75.40 \%$ \\
VGG-19 & $95.79 \%$ \\
ResNet & $96.32 \%$ \\
\hline
\end{tabular}

As can be seen from Table 10, DNN has great advantages over the traditional GMM classifier, while the ResNet model achieves the best classification results.

In addition, the training speeds of the VGG model and the ResNet model were recorded respectively, as shown in Table 11. The methods were been simulated by MATLAB software on a workstation with an 8-core CPU (I7 9700K) and 16 GB RAM. In both cases, the loss function reached convergence and no significant changes occurred in the fifth iteration, which was taken as the cutoff time.

Table 11. Training speed of different classification models.

\begin{tabular}{cc}
\hline Classification Model & Training Time \\
\hline VGG-19 & $26 \mathrm{~min}$ \\
ResNet-50 & $16 \mathrm{~min}$ \\
\hline
\end{tabular}

It can be seen from Table 11 that since ResNet can learn preload parameters through migration, the amount of training is reduced, so the training time is also significantly reduced. For each $2 \mathrm{~s}$ data frame, the total time of feature extraction and ResNet classification is about $16 \mathrm{~ms}$.

\subsubsection{Adaptability to New Samples}

In the previous experiment, the training set was constructed by randomly selecting $80 \%$ of the feature samples. The training samples involved records from all 90 ships. However, in the actual work of the classifier, it is possible to encounter recorded data of new vessels, and the sample obtained from this record is never seen by the classifier. To evaluate the classification performance of the classifier on new record data, we restricted the training set construction by taking feature samples corresponding to the $80 \%$ of recorded data as training samples, and the remaining $20 \%$ of feature samples as test samples. The construction methods of the two training sets are shown in Figure 17.

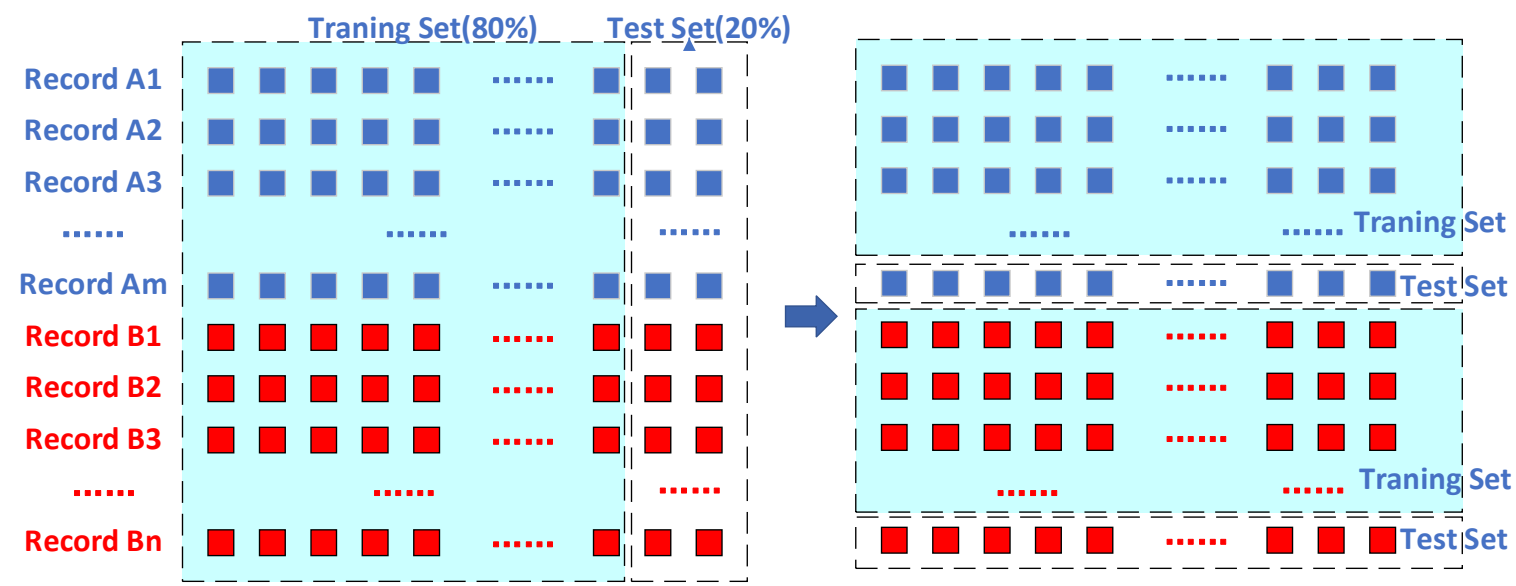

Figure 17. Two construction methods for the training set and test set: (left) random, (right) according to vessel records.

It can be seen from Table 12 that the results of the classification system have decreased, but the classification accuracy of $92.91 \%$ is acceptable. This indicates that the system has good adaptability to new samples. 
Table 12. Accuracy of different classification models.

\begin{tabular}{cc}
\hline Training Set Construction Method & Accuracy \\
\hline Random & $96.32 \%$ \\
According to vessel records & $92.91 \%$ \\
\hline
\end{tabular}

\section{Conclusions}

In this paper, a UATR method is proposed that uses MWSA to reduce the dimension of data. Spectrograms with different T-F resolutions are calculated through three windows to synthesize a 3-channel color spectrogram as the classification feature into the classification network. Combining the advantages of CGAN and DCGAN, the CDCGAN model is designed to realize the effective augmentation of samples. The designed classification network based on ResNet gives full play to the advantages of the residual module and can realize signal classification with high efficiency and high performance. According to the experiment based on the ShipsEar database, the accuracy of the proposed method is $96.32 \%$, which is a better classification performance than other current methods. The proposed method provides good technical support for the target classification and recognition function of a SONAR system. The performance test results of the proposed method are based on experiments with high-SNR data sets. The ship-radiated noise data obtained by actual SONAR systems usually have low SNRs and low signal-to-interference ratios (SIR). Feature extraction and data augmentation using the proposed method under a small sample set with low-SNR samples are worthy of further study.

Author Contributions: Conceptualization, X.L. and M.Z.; methodology, X.L., M.Z. and X.X.; software, M.Z.; validation, M.Z., T.L. and M.H.; formal analysis, X.L. and M.Z.; investigation, M.Z.; resources, X.L.; data curation, M.Z. and T.L.; writing—original draft preparation, M.Z., M.H. and T.L.; writingreview and editing, X.L. and M.Z.; visualization, X.L., M.Z. and X.X.; supervision, X.L.; project administration, X.L.; funding acquisition, X.L. All authors have read and agreed to the published version of the manuscript.

Funding: This research was funded by the National Natural Science Foundation of China under grant number 12174053, 91938203, 11874109, 11674057 and the Fundamental Research Funds for the Central Universities No. 2242021k30019.

Institutional Review Board Statement: Not applicable.

Informed Consent Statement: Not applicable.

Data Availability Statement: Not applicable.

Conflicts of Interest: The authors declare no conflict of interest.

\section{References}

1. Akyildiz, I.F.; Pompili, D.; Melodia, T. Underwater acoustic sensor networks: Research challenges. Ad Hoc Netw. 2005, 3, 257-279. [CrossRef]

2. Fang, S.; Du, S.; Luo, X.; Han, N.; Xu, X. Development of underwater acoustic target feature analysis and recognition technology. Bull. Chin. Acad. Sci. 2019, 34, 297-305.

3. Erol-Kantarci, M.; Mouftah, H.T.; Oktug, S. A survey of architectures and localization techniques for underwater acoustic sensor networks. IEEE Commun. Surv. Tutor. 2011, 13, 487-502. [CrossRef]

4. Luo, X.; Feng, Y. An underwater acoustic target recognition method based on restricted Boltzmann machine. Sensors 2020, 20, 5399. [CrossRef] [PubMed]

5. Luo, X.; Feng, Y.; Zhang, M. An underwater acoustic target recognition method based on combined feature with automatic coding and reconstruction. IEEE Access 2021, 9, 63841-63854. [CrossRef]

6. Zhang, L.; Wu, D.; Han, X.; Zhu, Z. Feature extraction of underwater target signal using mel frequency cepstrum coefficients based on acoustic vector sensor. J. Sens. 2016, 2016, 7864213. [CrossRef]

7. Khishe, M.; Mohammadi, H. Passive sonar target classification using multi-layer perceptron trained by salp swarm algorithm. Ocean Eng. 2019, 181, 98-108. [CrossRef]

8. Hu, G.; Wang, K.; Peng, Y.; Qiu, M.; Shi, J.; Liu, L. Deep learning methods for underwater target feature extraction and recognition. Comput. Intell. Neurosci. 2018, 2018, 1214301. [CrossRef] [PubMed] 
9. Qiao, W.; Khishe, M.; Ravakhah, S. Underwater targets classification using local wavelet acoustic pattern and Multi-Layer Perceptron neural network optimized by modified Whale Optimization Algorithm. Ocean Eng. 2021, 219, 108415. [CrossRef]

10. Biernacki, C.; Celeux, G.; Govaert, G. Assessing a mixture model for clustering with the integrated completed likelihood. IEEE Trans. Pattern Anal. Mach. Intell. 2000, 22, 719-725. [CrossRef]

11. Chang, C.C.; Lin, C.J. LIBSVM: A library for support vector machines. ACM Trans. Intell. Syst. Technol. 2011, 2, 1-27. [CrossRef]

12. Khishe, M.; Mosavi, M.R. Improved whale trainer for sonar datasets classification using neural network. Appl. Acoust. 2019, 154, 176-192. [CrossRef]

13. Stewart, W.K.; Jiang, M.; Marra, M. A neural network approach to classification of sidescan sonar imagery from a midocean ridge area. IEEE J. Ocean. Eng. 1994, 19, 214-224. [CrossRef]

14. Yue, H.; Zhang, L.; Wang, D.; Wang, Y.; Lu, Z. The classification of underwater acoustic targets based on deep learning methods. Adv. Intell. Syst. Res. 2017, 134, 526-529.

15. Hershey, S.; Chaudhuri, S.; Ellis, D.P.W.; Gemmeke, J.F.; Jansen, A.; Moore, C.; Plakal, M.; Platt, D.; Saurous, R.A.; Seybold, B.; et al. CNN architectures for large-scale audio classification. In Proceedings of the 2017 IEEE International Conference on Acoustics, Speech and Signal Processing (ICASSP), New Orleans, LA, USA, 5-9 March 2017; pp. 131-135. [CrossRef]

16. Krizhevsky, A.; Sutskever, I.; Hinton, G.E. ImageNet classification with deep convolutional neural networks. Commun. ACM 2017, 60, 84-90. [CrossRef]

17. Simonyan, K.; Zisserman, A. Very deep convolutional networks for large-scale image recognition. arXiv 2014, arXiv:1409.1556.

18. Doan, V.S.; Huynh-The, T.; Kim, D.S. Underwater acoustic target classification based on dense convolutional neural network. IEEE Geosci. Remote Sens. Lett. 2020, 2020, 1-5. [CrossRef]

19. He, K.; Zhang, X.; Ren, S.; Sun, J. Identity Mappings in Deep Residual Networks. In Proceedings of the European Conference on Computer Vision, Amsterdam, The Netherlands, 11-14 October 2016; Springer: Cham, Swizterland, 2016 ; pp. 630-645.

20. Perez, L.; Wang, J. The effectiveness of data augmentation in image classification using deep learning. arXiv 2017, arXiv:1712.04621.

21. Goodfellow, I.; Pouget-Abadie, J.; Mirza, M.; Xu, B.; Warde-Farley, D.; Ozair, S.; Courville, A.; Bengio, Y. Generative adversarial nets. In Advances in Neural Information Processing Systems; ACM Digital Library: New York, NY, USA, 2014 ; Volume 27.

22. Mirza, M.; Osindero, S. Conditional generative adversarial nets. arXiv 2014, arXiv:1411.1784.

23. Radford, A.; Metz, L.; Chintala, S. Unsupervised representation learning with deep convolutional generative adversarial networks. arXiv 2015, arXiv:1511.06434.

24. Santos-Domínguez, D.; Torres-Guijarro, S.; Cardenal-López, A.; Pena-Jimenez, A. ShipsEar: An underwater vessel noise database. Appl. Acoust. 2016, 113, 64-69. [CrossRef]

25. Hong, F.; Liu, C.; Guo, L.; Chen, F.; Feng, H. Underwater acoustic target recognition with a residual network and the optimized feature extraction method. Appl. Sci. 2021, 11, 1442. [CrossRef]

26. Donahue, C.; McAuley, J.; Puckette, M. Adversarial audio synthesis. arXiv 2018, arXiv:1802.04208.

27. Sharan, R.V.; Moir, T.J. Pseudo-color cochleagram image feature and sequential feature selection for robust acoustic event recognition. Appl. Acoust. 2018, 140, 198-204. [CrossRef]

28. Salamon, J.; Bello, J.P. Deep convolutional neural networks and data augmentation for environmental sound classification. IEEE Signal Process. Lett. 2017, 24, 279-283. [CrossRef] 